\title{
Cartografiar lo común: trabajo colaborativo e interdisciplinar para la resemantización territorial
}

\section{Mapping the common: collaborative and interdisciplinary work for territorial resematization}

\author{
Beatriz Cid ${ }^{1}$, Loreto Arias $^{2}$ (autora correspondiente), Isidora Troncoso ${ }^{3}$, Magaly Mella ${ }^{4}$, \\ Francisca Abarca ${ }^{5}$ y Karina Alveal ${ }^{6}$ \\ Recibido 15/09/2020. Aceptado 15/03/2021 \\ http://dx.doi.org/10.30972/dpd.10154806
}

\section{Resumen}

Este artículo presenta la técnica de la cartografía social como un instrumento que propicia la aproximación de mundos a través del uso y eficacia del trabajo colaborativo interdisciplinar y la efectiva participación en ejercicios de diseño conjunto y diálogo de saberes. En particular, este documento muestra la metodología usada en el aprendizaje colectivo y de vinculación territorial con diferentes comunidades del centro sur de Chile: campesinas e indígenas, dedicadas a la viñatería, a la agroecología, a la recolección de productos silvestres, al turismo comunitario y a la pesca artesanal en ríos. Con ellas, se llevaron a cabo ejercicios para documentar, promover y amplificar sus prácticas de producción de alteridad económica y de creación y cuidado de sus comunes. Las cartografías desarrolladas no reflejan datos positivistas, exactos y objetivos, sino representaciones, imaginarios, sentimientos, desafíos, logros y aspiraciones de quienes habitan esos territorios y contribuyen a la resemantización de los mismos para la ampliación de los horizontes políticos. Se reflexiona también sobre la relevancia de trabajar sinérgicamente entre ciencia y comunidades en procesos de colaboración y aprendizaje mutuo.

Palabras clave: resemantización; comunes; diversidad económica.

\footnotetext{
${ }^{1}$ Socióloga, Pontificia Universidad Católica de Chile, PhD Sociología York University. Profesora Asociada del Departamento de Sociología de la Universidad de Concepción. Beatrizcid@udec.cl

${ }^{2}$ Socióloga, Universidad de Concepción, Personal Técnico FONDECYT 1190020. Lariasr92@gmail.com

${ }^{3}$ Geógrafa, Magíster (c) en Investigación Social y Desarrollo, Universidad de Concepción. Isitroncoso@gmail.com

${ }^{4}$ Investigadora del Centro de Estudios Urbano Regionales de la Universidad del Bío-Bío. Antropóloga, DEA y Dra. (c) Programa de Doctorado en Antropología Social y Cultural, Universidad de Barcelona. Magmella@ubiobio.cl

${ }^{5}$ Licenciada en Sociología, Universidad de Concepción. Franciscaabll@gmail.com

${ }^{6}$ Licenciada en Sociología, Universidad de Concepción. Karialveal@gmail.com
} 


\begin{abstract}
This article highlights the technique of social cartography as an instrument that fosters the approximation of worlds, through interdisciplinary work, and effective participation in collective design exercises and dialogues of knowledge. In particular, this document shows the methodologies used in collective learning and territorial vinculation with different communities in the central South of Chile: peasant and indigenous, dedicated to vineyards, agroecology, the collection of wild products, community tourism and artisanal fishing in rivers. We developed exercises to document, promote and amplify their practices of economic alterity and the creation and care of commons. The participatory maps do not reflect positivist, exact, and objective data, but rather representations, imaginations, feelings, challenges, achievements and aspirations of the inhabitants, and contribute to the territorial resematization for the broadening of political horizons. The document also reflects on the relevance of synergistic cooperation between science and communities for mutual learning.
\end{abstract}

Keywords: resematization, commons, economic diversity.

\title{
1.- Introducción
}

Este artículo presenta la técnica de la cartografía social como un instrumento que propicia la aproximación de mundos a través del uso y eficacia del trabajo colaborativo interdisciplinar y el ejercicio de diseño conjunto y diálogo de saberes. Los mapas participativos materializan las representaciones clave de las diferentes realidades socioculturales, objetivándolos en mapas georreferenciados y cartografías ilustradas. Ello permite, sobre la base de una relación horizontal de intereses y posiciones transparentadas, alcanzar no solo hallazgos científicos y nuevos conocimientos, sino fortalecer capacidades locales con técnicas dialógicas y de vinculación entre diferentes personas. Así también, permite dotar de sólidos fundamentos para la salvaguardia y proyección de las comunidades.

El texto documenta, particularmente, un proceso de aprendizaje colectivo, interdisciplinar y de vinculación territorial con diferentes comunidades del centro sur de Chile, entre ellas, comunidades campesinas dedicadas a la viñatería y a la agroecología en el valle del Itata de la región del Ñuble, el Maule y Valparaíso; la recolección de productos silvestres de la comuna de Empedrado de la región del Maule; el turismo comunitario e indígena del Alto Biobío y Tirúa de la región del Biobío, y la pesca artesanal en el río Toltén de la Araucanía, con quienes se llevaron a cabo ejercicios de cartografía social para documentar, promover y amplificar sus prácticas de producción de alteridad económica y de creación y cuidado de bienes comunes. Los ejercicios de cartografía social tuvieron como objetivo destacar la 
diversidad económica de los territorios, los bienes comunes que les subyacen, las tragedias y el despojo que han sufrido, y las diversas formas de gobernanza que ejercen las comunidades en escenarios cada vez más complejos. A través de procesos dialógicos llevados a la práctica en una vinculación territorial responsable, donde prima la colaboración y el intercambio de conocimientos y experiencias, se construyó un diálogo de saberes que permitió la aproximación de mundos y la construcción conjunta de lo social.

Este trabajo busca destacar la relación que establecen las comunidades con su territorio, dibujando en forma participativa las claves y las alternativas existentes para la gobernanza de sus comunes. Se trata de una metodología inspirada en el potencial relacional entre ciencia y comunidades, donde ambas tienen mucho que aprender y colaborarse mutuamente para la transformación de sus propias realidades. En sí misma, la técnica de cartografía social permite territorializar los recursos, las amenazas y las estrategias que utiliza la comunidad, propiciando una reflexión conjunta en la elaboración de un diagnóstico coconstruido. Los territorios obtienen un insumo para su quehacer colectivo, visibilizando un conjunto de aspectos constitutivos.

Las cartografías desarrolladas fueron sistematizadas de forma interdisciplinaria, dando lugar a mapas georreferenciados e ilustrados con los elementos identificados por la propia comunidad. De esta forma, los mapas no tienen la pretensión de ser resultados positivistas, exactos, objetivos de los lugares, sino que buscan reflejar sentimientos, desafíos, logros y sueños de quienes habitan aquellos territorios.

\section{2.- Cartografía social: procesos de resemantización de lo económico y común en los territorios}

La cartografía social es una metodología participativa de investigación que permite a las comunidades reflexionar y poner en común los conocimientos sobre el espacio que habitan:

Se trata de construir desde la reflexión y la discusión de la situación problematizadora, espacios, redes, el territorio en el que se intercambian saberes, solidaridades y de esta forma legitimarlos desde sus aportes al accionar. En definitiva, detrás de los mapas no hallamos solamente fronteras, símbolos, colores, líneas, realidades inconexas, sino trayectos, recorridos, movimientos, intercambios y complejos procesos y creatividades que hacen visible un mejor vivir. (Habegger y Mancila, 2006: 3)

Esto se logra a través de la elaboración conjunta de mapas, que desata importantes procesos de comunicación entre sus participantes y pone en diálogo múltiples saberes, prácticas y experiencias que al encontrarse permiten llegar a una imagen colectiva (Andrade y Santamaría, 1997; Diez y Chanampa, 2016). Lo anterior resalta otras formas de concebir y habitar los espacios, distintas a las establecidas en la perspectiva cartográfica tradicional: 
Asumiendo esta perspectiva, podemos problematizar la relación entre territorio y producción de conocimiento, reconociendo con ello que el saber es la gente y el espacio que habita, más que un mero depósito de información sobre el cual se realizan análisis, siendo los pobladores más bien agentes activos con quienes se interactúa y se producen conjuntamente conocimientos desde sus territorios. (Montoya, Sánchez y Ospina, 2014: 5)

Como método, la cartografía social es parte y heredera de la Investigación Acción Participativa, que trabaja íntimamente ligada a la vida social en clave emancipatoria (Ángel Pérez, 2011), en compromiso ético de cocreación con la comunidad, "reconociendo que el conocimiento popular puede ser congruente con la heredada de la ciencia académica" (Fals Borda, 2009: 362).

En América Latina se han desarrollado diversas experiencias de cartografía social. En Colombia, comunidades indígenas y afrodescendientes han desarrollado procesos de autoinvestigación, recuperación cultural y educación denominados "mapas parlantes", que depositan los conocimientos de la gente y los aportes de la investigación científica con el propósito de elaborar una herramienta pedagógica para el territorio en el contexto de conflictos socioambientales y luchas por la tierra (Montoya, Sánchez y Ospina, 2014). Por su parte, el grupo Iconoclasistas, conformado por la Dra. Julia Risler y el artista visual Pablo Ares, trabaja desde 2008 la investigación colectiva con el mapeo creativo y el arte gráfico para la investigación colaborativa con colectivos en procesos de lucha territorial. Otra experiencia en este ámbito es la Cátedra libre de cartografía social, la cual convoca tres grupos de trabajo (La Plata y Comodoro Rivadavia en Argentina y Pelotas en Brasil) para mapear lo invisible bajo la influencia teórica de Deleuze y Guattari, la pesquisa cartográfica de Kastrup y Passos, la teoría de la deriva de Debord y la propuesta de intervención social de Alfredo Carballeda.

Se comprende la cartografía como una intertextualidad entre el mapa, el debate, la reflexión y el intercambio de experiencias, contribuyendo a crear una nueva realidad. La premisa inicial es que todos somos productores de territorio; en consecuencia, el mapeo se realiza sin mesas y sin mapas, para emancipar el discurso de las medidas cartográficas tradicionales, elaborando "derroteros" que proponen los cartografiables desde el territorio y la comunidad. Así, la experiencia generada a partir del intercambio genera un nuevo plano común de perspectivas y realidades que queda plasmado en el mapa (Diez y Chanampa, 2016). Por último, el Proyecto Nova Cartografia Social da Amazonia (PNCSA) convoca a investigadores de las ciencias naturales y sociales en Brasil para potenciar la autocartografía de pueblos indígenas del Amazonas sobre la ocupación del territorio y su manifestación en identidades colectivas territoriales, promoviendo así procesos de territorialización.

Nuestro trabajo encuentra en la cartografía social una metodología de la intertextualidad para la resemantización económica y política de los territorios. El propósito de resemantizar 
se inspira en el trabajo de Callon (2007), Butler (1990) y, especialmente, de Gibson-Graham (2006). Resemantizar es un elemento central para elaborar guiones y proyectos alternativos, ampliando el universo de posibilidades (Gibson-Graham, 2006) para tensionar los relatos y cartografías hegemónicas que condicionan nuestra imaginación territorial y contribuyen a limitar el universo económico y político de los territorios. Cuando la teoría neoclásica ha elevado estos relatos a lenguaje científico matemático, contribuye a modelar la acción de los agentes económicos territoriales, así como también sus procesos políticos (Callon, 2007). Los relatos cartográficos dominantes son estadocéntricos y capitalocéntricos (Gibson-Graham, 2006), como tal, contribuyen a invisibilizar las relaciones económicas diversas de los territorios constituyendo una forma de violencia sobre otras expresiones de economía limitando la generación de discursos transformadores. Este enfoque apunta a la capacidad del lenguaje para redefinir no solo los discursos, sino nuestra imaginación económica, nuestras visiones de futuro (Gibson-Graham, Cameron y Healy, 2017) y nuestros territorios. Arturo Escobar (2017: 108) señala que "descentrar a la economía de la vida social y ecológica es una condición especial de todo activismo y diseño para la transición".

El enfoque de economías diversas que nos inspira (Gibson-Graham, 2006) reconoce que la economía está constituida por diversas formas organizativas y que los actores económicos responden a variadas lógicas de valoración y acción. Como tal, reexamina las prácticas sociales para dar cuenta de su pluralidad y, por lo tanto, disminuir la hegemonía capitalocéntrica permitiendo la afirmación de alternativas (Gibson-Graham et al., 2017), donde las personas están activamente involucradas en recuperar el control sobre los procesos económicos (Eskelinen, Hirvilammi y Venäläinen, 2020). Reconoce también que la reproducción de la vida (Carrasco, 2001; Pérez Orozco, 2004) y de lo comunal (Escobar, 2017) se escapa del discurso y, más aún, de la cuantificación de lo tradicionalmente considerado económico. Los mapas, y su proceso de elaboración, pueden ser espacios para mirar la vida económica y política del territorio, descubriendo retrospectivamente otras inscripciones en el mismo, de procesos económicos antes invisibles y desplazados por el capitalocentrismo dominante.

Lo comunal se comprende en el sentido de Gibson-Graham et al. (2017: 168), "al definir a un común como una propiedad, una práctica o un conocimiento que es compartido por una comunidad". La visión que se propone convoca a entenderlos no como un conjunto delimitado de bienes, sino, más bien, como el resultado de prácticas políticas colectivas y generativas que los crean, cuidan y expanden permanentemente. La existencia de bienes comunes -desde la naturaleza hasta los saberes- está anclada en sujetos comunitarios que los construyen, gestionan, protegen y amplían frente a los sucesivos cercamientos privatizadores (Hardt y Negri, 2011), despojos (Harvey, 2004) y tragedias por mal uso (Hardin, 1968). La cartografía social como metodología permite destacar tanto los comunes 
compartidos -que para la comunidad son fundamentales- como también lo relativo a los cercamientos, tragedias y despojos que los amenazan y, por consecuencia, a la comunidad. En este contexto, se documenta la gobernanza y las estrategias utilizadas frente a las amenazas y las transformaciones que podrían sufrir los comunes. Laval y Dardot (2014: 170) lo señalan como "la necesidad de las diversas reglas y prácticas que permiten producir y reproducir recursos comunes", materializadas en "normas y reglas sociales, así como mecanismos legales que permiten a individuos compartir la propiedad y el control de los recursos". En último caso, la gobernanza se sintetizaría en la relación consciente que establece la comunidad con el común.

En suma, se utiliza la cartografía social para ser parte de la tradición de Investigación Acción Participativa, con el propósito de contribuir a una resemantización económica y política de los territorios y de la economía comunalizadora de la vida, que permite imaginar juntos otros futuros territoriales.

\section{El ejercicio metodológico}

La metodología se desarrolla en tres momentos que corresponden a (1) la preparación de la cartografía, (2) su elaboración y finalmente -como un paso central- (3) su validación comunitaria. Cada uno de estos tres momentos se llevan a cabo en articulación y coordinación con las comunidades. Si bien se habla de tres momentos metodológicos, debemos señalar que este trabajo se ha realizado con colectivos y comunidades con los cuales existe una relación de confianza y colaboración de mediana y larga data, lo que se considera como condición previa para el desarrollo de los mismos.

El objetivo general de la metodología que se expone es identificar y caracterizar participativamente los comunes que sustentan las actividades económicas y sociales de un territorio, como también la existencia de procesos de tragedia y despojo, reconociendo los ejercicios de gobernanza desplegados por las comunidades para un diseño territorial autónomo.

\section{Momento 1: preparación de la cartografía}

Para este momento se desarrollan a lo menos dos encuentros. En un primer encuentro, los/as dirigentes/as se familiarizan con la metodología y se dialogan los objetivos de investigación, de modo que estos contribuyan a los procesos políticos y organizacionales de la comunidad, cocreando la información. A partir de esta reunión, los dirigentes evalúan con sus comunidades la realización de la cartografía social. Luego de ello, se lleva a cabo un conjunto de encuentros que definen aspectos técnicos y logísticos relevantes, tales como: 
- Definición de superficie, escala del mapa y diacríticos referenciales (tales como caminos, escuelas, paraderos de autobús, iglesias, entre otros): estos permiten a las personas ubicarse en el territorio y su reconocimiento en el mapa. Esto es muy relevante, puesto que muchos de los participantes nunca han trabajado previamente con mapas.

- Delimitar al grupo asistente: establecer el número de personas asistentes y además conocer sus cualidades, tales como la edad, género, oficios, relación con el territorio, etc. Esto es muy importante pues, si tenemos un grupo de personas adultas mayores, podemos anticiparnos a pedirles durante la convocatoria que acudan con sus anteojos o en caso de analfabetismo, adecuar nuestras estrategias de facilitación.

- Otros aspectos logísticos: seleccionar y gestionar un lugar adecuado (como la sede vecinal), el día y la hora para el ejercicio, apoyar en la convocatoria a la actividad y preparar los materiales para el ejercicio, tales como la impresión del mapa base, papelógrafos, papel diamante o vegetal para las capas de la cartografía, lápices de colores, stickers con símbolos que tengan pertenencia cultural con el contexto y grabadoras de audio o cámaras para el registro.

\section{Momento 2: elaboración de la cartografía}

La elaboración de las cartografías involucra dos o más jornadas de trabajo colectivo para discutir y dibujar colectivamente los mapas. Se recomienda que las jornadas de trabajo no excedan las 3 horas y que existan espacios de descanso y recreación. Durante el primer encuentro se aplican el punto 1 y 2 , descritos a continuación, mientras que durante el segundo encuentro se problematizan el punto 3 y 4.

Preparar el ejercicio implica, en primer lugar, adecuar el espacio -la sede vecinal o comunitaria o el sindicato- verificando la disponibilidad de mesas y sillas para el trabajo en grupos. Dependiendo del número de asistentes, es necesario evaluar cuántos subgrupos de trabajo se crearán para promover la participación; se sugiere conformar grupos de entre 7 y 10 personas. De esta manera, se deben asegurar al menos dos facilitadores para cada grupo de trabajo. La dupla de facilitadores tiene el rol de dirigir la conversación, moderar, elaborar las preguntas detonantes y verificar que la reflexión colectiva quede consignada en el mapa. También es parte de su rol asistir a quien lo necesite, controlar los tiempos de cada actividad y verificar el registro de la jornada a través de fotos o grabadoras.

1. Primer ejercicio cartográfico 
El ejercicio cartográfico comienza con la presentación del equipo de facilitadores y los participantes, para lo cual se recomienda utilizar una dinámica "rompe hielo". Así también, es necesario lograr que los asistentes se familiaricen con la técnica, lo cual no es siempre fácil, pues en algunas comunidades nunca han visualizado su lugar proyectado en un espacio cartesiano. Para lo anterior, el equipo técnico presenta un mapa impreso a los asistentes y se les pide que ubiquen sus casas, sus actividades económicas y sus productos, así como también los elementos que consideren importantes tales como los caminos, los ríos, empresas, poblados, mar, sedes sociales, etc. Como resultado, se crea un mapa intervenido con los elementos relevantes y referenciales del territorio desde la perspectiva de la comunidad.

2. Identificación e historicidad de los comunes

Luego de esa primera intervención cartográfica, se desarrolla un taller que busca identificar los comunes presentes en el territorio. Para ello, se realizan las siguientes preguntas: ¿Qué elementos naturales o culturales, recursos, riquezas del territorio son compartidos por todos ustedes y son fundamentales para el desarrollo de sus actividades?

A partir de aquí, se elabora una lista de elementos que son anotados en un papelógrafo. Usualmente surgen elementos materiales, tales como el piñón, carbón y el agua; sin embargo, también pueden surgir elementos inmateriales, como los saberes y conocimientos, la solidaridad, la unión y la organización. A partir de la lista se invita a los participantes a ubicar estos elementos en los mapas, utilizando adhesivos, símbolos, el dibujo o la escritura. De esta manera se obtiene como resultado un mapa de la visión de la comunidad sobre los comunes territorializados.

Figura 1. Mapa intervenido de la comuna del Valle del Itata 


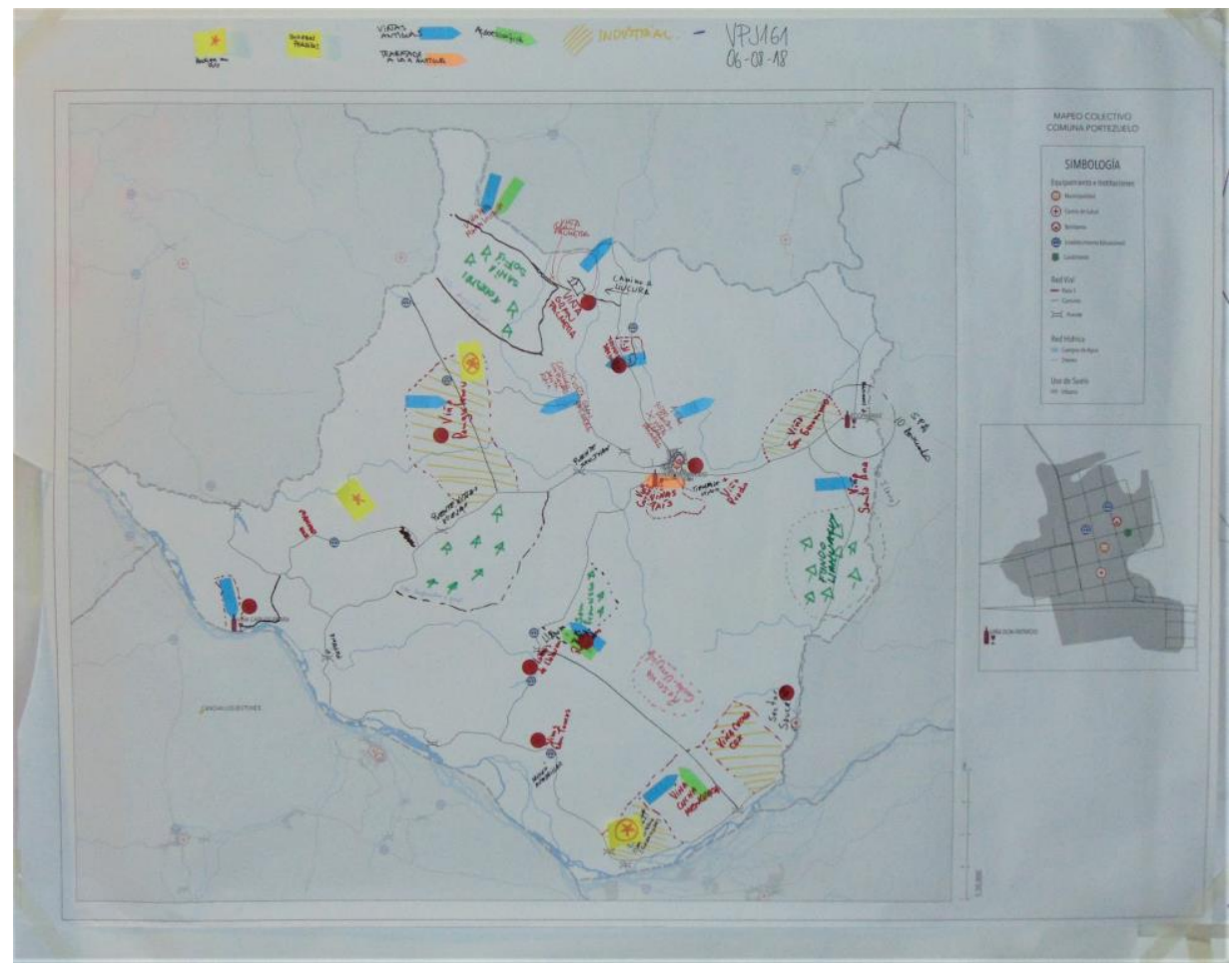

Fuente: Proyecto Fondecyt 1160186 y 1190020

Este ejercicio abre una discusión en torno a la historicidad del común con discusiones alrededor de preguntas tales como ¿de dónde vienen estos comunes? ¿Desde cuándo se valoran? ¿Desde cuándo la comunidad reconoce este recurso como algo importante de defender? ¿Cómo lo reivindicaron? Toda esta reflexión destaca el proceso de reconocimiento y creación del común como ejercicio de actividad comunitaria.

3. Tragedias y despojos del común

Luego de identificar los comunes, un tercer ejercicio cartográfico abre la reflexión sobre los procesos de apropiación -privatización y despojo- y deterioro o tragedia de los comunes. Para ello, se propone un segundo encuentro utilizando el mapa producido en el primero, pero en torno a preguntas tales como ¿qué amenazas tienen los comunes enlistados anteriormente? ¿Alguien se quiere apropiar de estos recursos territoriales? ¿Quiénes? ¿Qué problemas de uso o aprovechamiento de estos pueden estar deteriorándolos? Las respuestas se van relacionando con los comunes que se enlistaron en la sección anterior. Por ejemplo, si el "mar" es el común, se profundiza en quiénes se lo apropian y qué actividades o actores lo deterioran. Finalmente, se invita a ubicar estas amenazas dentro del mapa, en una nueva capa que corresponde a las tragedias y los despojos de los comunes. Para ello, se usan símbolos, dibujos y textos explicativos, logrando al final de esta actividad un mapa con el despojo y tragedia de los comunes.

4. Gobernanza del común 
Luego de identificados los comunes territoriales, así como las tragedias y despojos que les afectan, la última capa del mapa pone énfasis en los ejercicios de defensa, cuidado y gobernanza del común. Esto es, por una parte, los ejercicios de lucha desarrollados para la reinvindicación de los comunes, como también las reglas acordadas que regulan el acceso o uso del común y promueven su cuidado y/o expansión. Para lo anterior, se facilita una discusión en torno a las tragedias y despojos antes documentados a través de la siguiente pregunta: ¿Qué acciones colectivas hace la comunidad para defender y cuidar -preservar o ampliar y fortalecer- el común? Al igual que en las secciones anteriores, se enlistan las conclusiones en un papelógrafo. Muchos de los elementos que emergen tendrán relación con las normas, los límites, el cuidado, proyectos o actores clave en torno a los comunes. Posteriormente, se localizan en el mapa los elementos surgidos, a través de símbolos, dibujos y textos. Al finalizar, obtendremos un mapa de los procesos de lucha, negociación, reglamentos y acuerdos de la comunidad, así como también se relevarán aquellos elementos de conflictos que aún no han sido abordados por el grupo.

Figura 2. Trabajo sobre capas

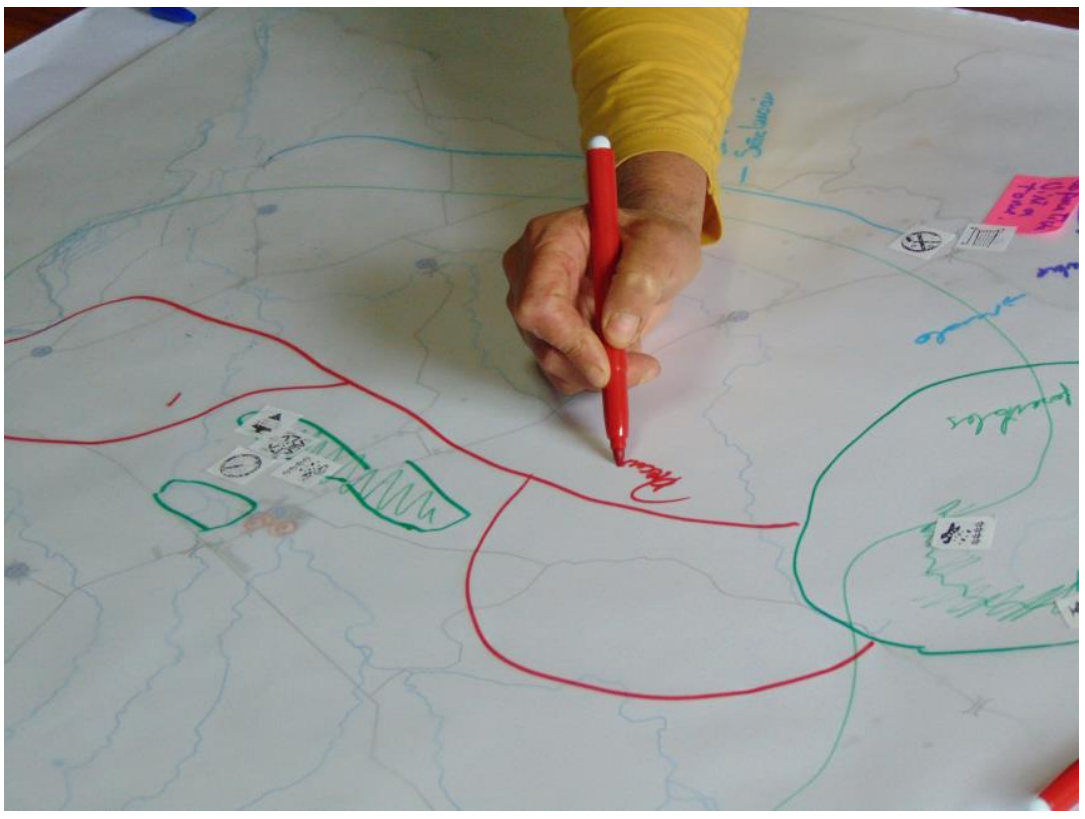

Fuente: Proyecto Fondecyt 1160186 y 1190020

Finalmente, para cerrar el proceso cartográfico, se invita a los y las participantes a reflexionar en torno a los desafíos que releva la cartografía, con preguntas tales como: ¿Qué 
aprendimos sobre la defensa y el cuidado de nuestros comunes? ¿Qué desafíos nos quedan? De lo que hemos hecho, ¿qué puede ser útil para otras comunidades?

\section{Momento 3: sistematización interdisciplinaria y validación(es) de la cartografía}

El procesamiento y sistematización del material producido por la comunidad comienza durante el mismo proceso cartográfico. En los espacios de descanso entre los distintos talleres, el equipo técnico y líderes comunitarios hacen una primera síntesis y ordenamiento de las discusiones para ser presentadas en el taller siguiente. Posteriormente, esta primera sistematización es profundizada, haciendo una transcripción literal de las discusiones, georreferenciando los puntos e hitos identificados y triangulando con otras fuentes de información. Durante todo el proceso de sistematización, se realizan reuniones periódicas con la comunidad, para compartir propuestas de avance y enfoques, corrigiendo de forma participativa los mismos. Como resultado de esta primera parte del análisis, se elabora un mapa georreferenciado que sintetiza los elementos emergidos durante la cartografía y un informe analítico. La figura $\mathrm{N}^{\circ} 3$ muestra un acercamiento a una zona que poseía gran cantidad de comunes.

Figura 3. Mapa georreferenciado 


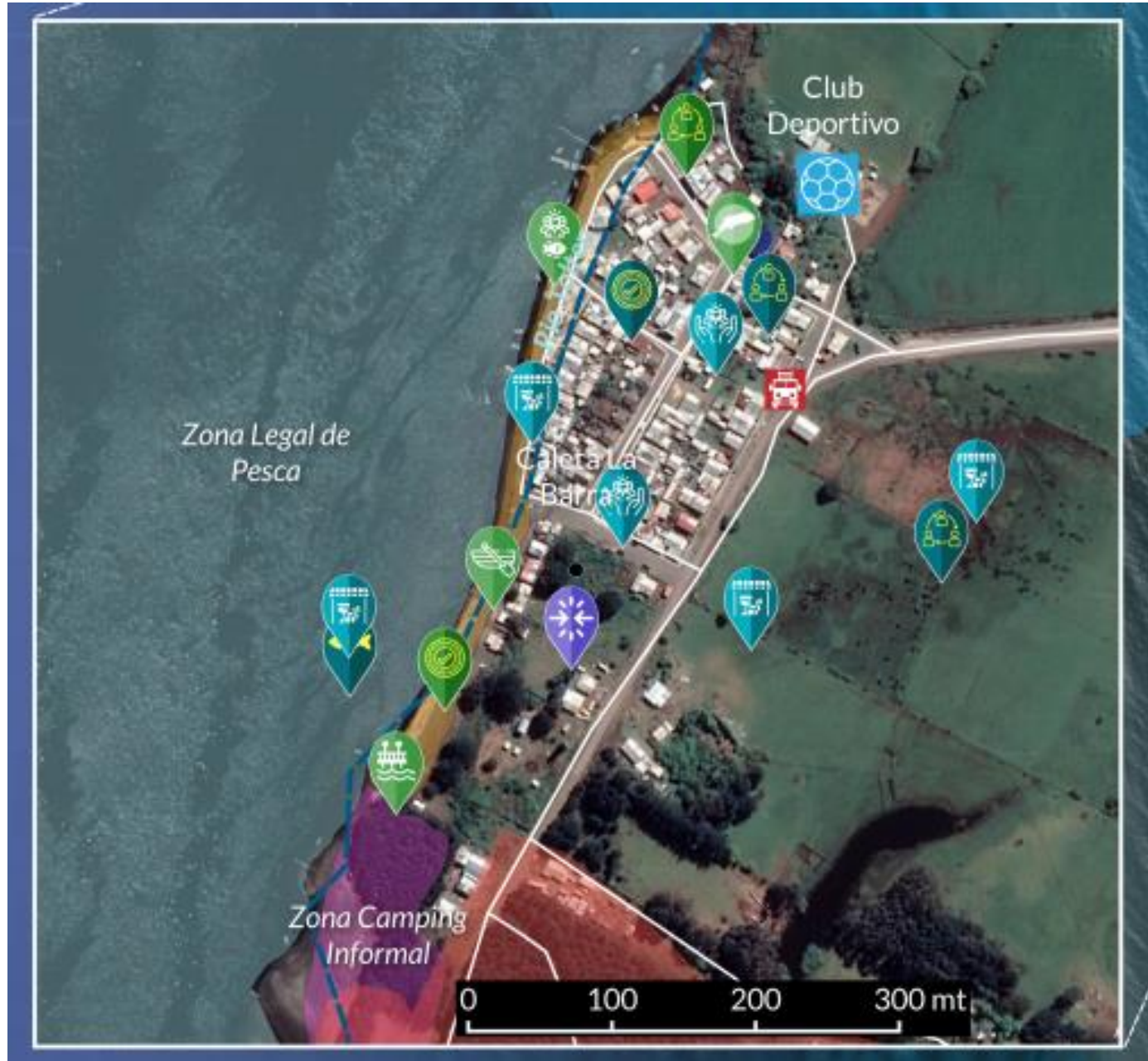

Fuente: Proyecto Fondecyt 1160186 y 1190020

Para esta primera parte del análisis, se organiza la información de los mapas en torno a las siguientes categorías: a) elementos referenciales de importancia para la comunidad, que son parte del uso cotidiano del paisaje y forman parte del mapa base; b) comunes identificados; c) despojo y tragedia; y d) gobernanza. Además, en este momento, se busca rescatar el discurso y los significados de la comunidad (contenido en las grabaciones transcritas del proceso cartográfico), vinculando el habla -textual- con los símbolos e hitos topográficos consignados, los que quedan incluidos en el mapa bajo la forma de leyenda. Así, cada elemento consignado en el mapa es acompañado de una definición situada y emanada de la comunidad, un ejemplo a continuación:

El Lance: "Cuando sacábamos harta corvina, se llamaba al 'lance'. Había como 4 a 5 viejos tirando redes, entonces ahí se metían viejitos adentro de la mar en un bote a encerrar los pescados. Lo encerraban con unos cordeles largos, una punta, y con el bote se iba dando la vuelta y salía la otra punta. Ahí iba toda la población a tirar la red para fuera. Aquí toda la 
población ganaba con el lance, el dueño de la red, el bote y quienes dirigían el lance ganaban más, luego seguían las mujeres y los niños [...] todos ganaban. Así se trabajaba antes, eso se llama unión. (Pescador Caleta La Barra, comunicación personal).

Figura 4. Mapa ilustrado Caleta La Barra

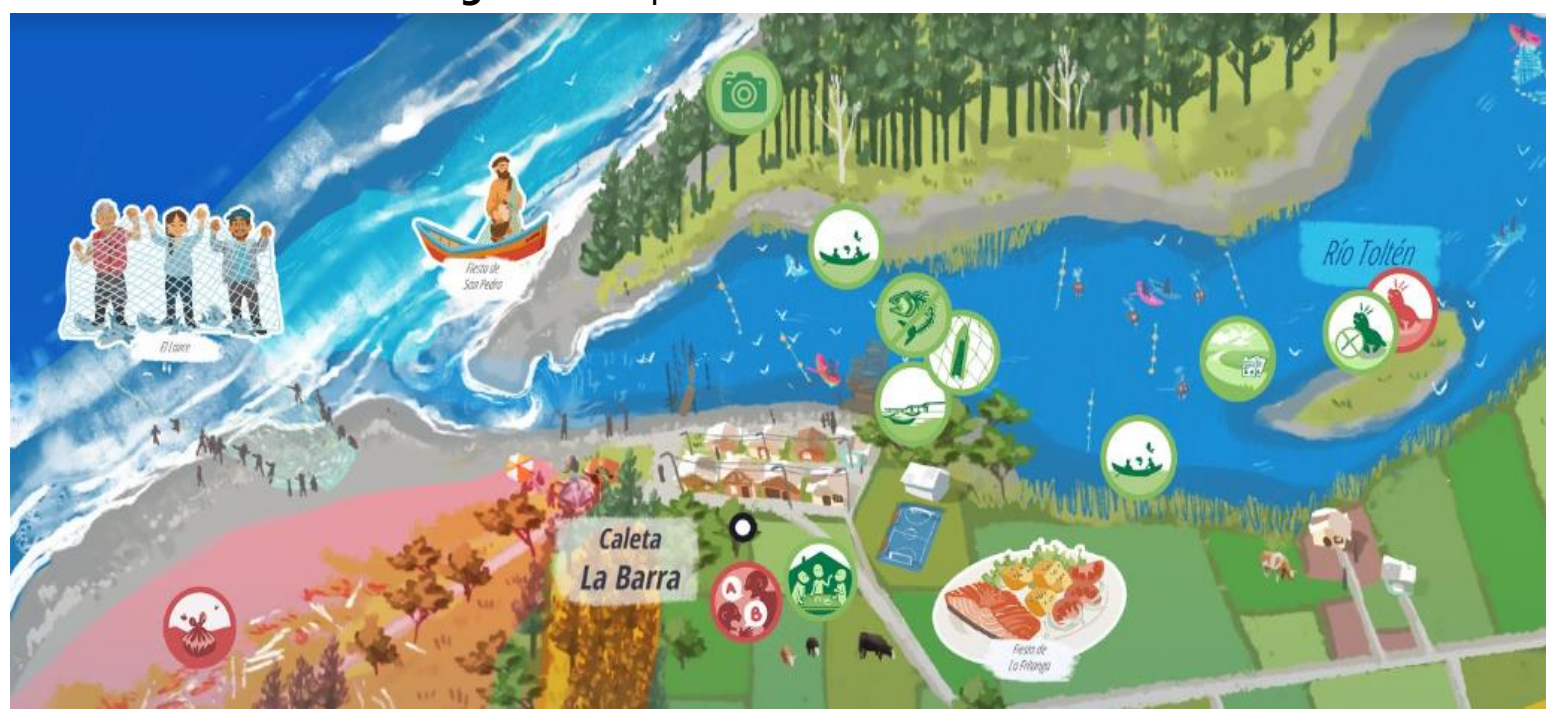

Fuente: Proyecto Fondecyt 1160186 y 1190020

Un segundo momento analítico busca llevar este mapa cartográfico a un mapa ilustrado, que con las herramientas del arte busca contener y expresar los discursos, subjetividades e imaginaciones del territorio. Este mapa es elaborado por un ilustrador que trabaja en forma conjunta con la comunidad para definir la estética, símbolos, colores y dibujos que sean pertinentes y adecuados al contexto. Esto es especialmente relevante, pues es necesario adecuar estéticas y símbolos, muchas veces urbanos y occidentales, a las estéticas y significados propiamente locales. Una anécdota que refleja esta tensión es ilustrar ciertos productos locales, como la avellana chilena, por ejemplo, como una avellana europea.

En nuestra experiencia, durante la sistematización se desarrolla una nomenclatura que permite el diálogo interdisciplinar. Desde la geografía, los elementos surgidos durante la cartografía se constituyen como puntos, polígonos y líneas que dan lugar a un mapa con vista satelital y georreferenciado. Por su parte, la ilustración representa los elementos surgidos a partir del sentido que le otorgan los habitantes, para lo cual, los divide en hitos, íconos y textura. Esta diferenciación de elementos está dictada por su repetición en el territorio y es un diálogo interdisciplinario entre la representación geográfica y la representación visual (como se muestra en la Tabla 1) que debe ser permanentemente validada por la comunidad. 
Tabla 1. Nomenclatura interdisciplinar

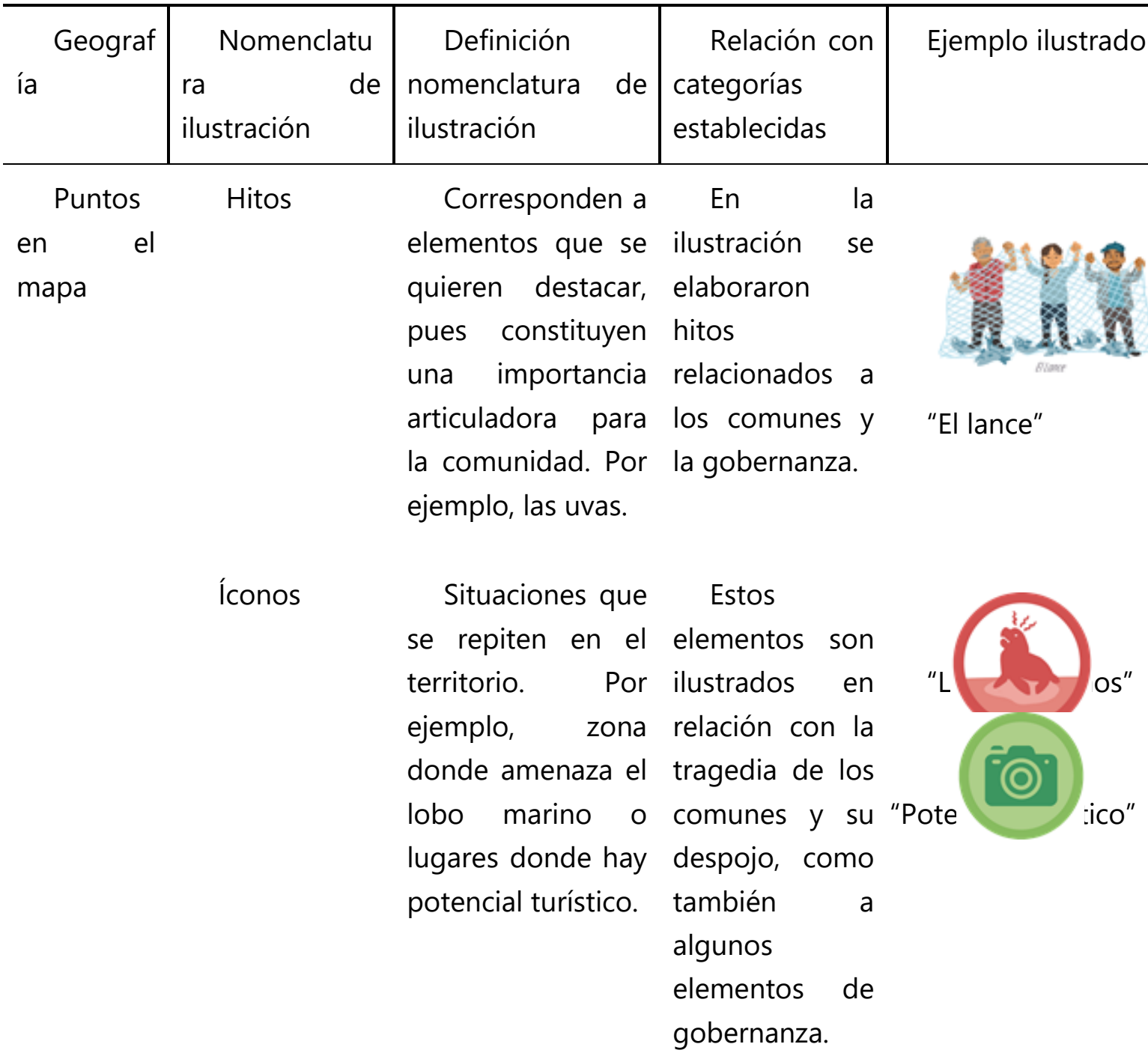

Polígon Texturas Zonas de gran Las áreas OS $\begin{array}{lll}\text { extensión } & \text { e } & \text { son } \\ \text { importancia para } & \text { representadas }\end{array}$ la comunidad. en la ilustración Ejemplo: áreas de con un filtro de pesca o zonas color lo "Zona forestal" forestales. corresponden a usos de suelo extensivo a lo 
largo y ancho

del territorio.

Fuente: Proyecto Fondecyt 1160186 y 1190020

A diferencia de otras técnicas grupales de levantamiento y análisis de información, la cartografía social utiliza como soporte una representación cartográfica del territorio, entregando un material que facilita la vinculación con los participantes. Dicho de otro modo, en los ejercicios de cartografía social, la comunidad observaba su territorio, el lugar que habita y construye, trazado en un papel y logra identificarse a partir de ello. El mapa facilita la participación al generar interés en la comunidad mediante la representación de algo propio y, también, por ser un medio didáctico y amigable. Cabe destacar que, tanto el dibujar como el escribir, puede generar resistencias en las personas por los juicios sociales que existen en torno a estas formas de expresión (el "saber" dibujar, el "dibujar bien", tener "linda letra"), por lo que propiciar un espacio de confianza, seguridad y contención es muy importante para estas instancias.

\section{Ajustes por pandemia}

Hemos realizado este ejercicio de cartografía social en diversas comunidades entre 2016 y 2020; sin embargo, algunos procesos se han interrumpido debido a la situación de pandemia. Originalmente, realizamos todos los momentos de la metodología de forma presencial; no obstante, correspondiendo al compromiso que tenemos con la salud de las comunidades y del equipo de investigación, decidimos prevenir los contagios evitando aglomeraciones, resolviendo trabajar principalmente con plataformas telemáticas y solamente con actores clave del territorio. Este escenario imprevisto plantea desafíos relacionados a la alfabetización digital de la comunidad y la infraestructura presente en el lugar que permita acceder a redes digitales. Estas circunstancias nos invitan a flexibilizar y buscar de forma creativa cómo llevar a cabo estos procesos manteniendo la participación. Un elemento interesante que la situación de pandemia ha permitido es el traslado de muchas de las responsabilidades en torno a la recopilación de datos y validación, desde el equipo de investigación a la propia comunidad. Es así como dirigentes de las comunidades especialmente los más jóvenes- adquirieron un rol cada vez más protagónico en el proceso 
local de investigación, recayendo en ellos gran parte del proceso de validación. En el actual contexto de inestabilidad sanitaria, sería también interesante avanzar en ejercicios móviles, que no implicaran reuniones en espacios cerrados, que favorezcan visitar los lugares. Hasta ahora, nuestro trabajo ha privilegiado el diálogo, la memoria y el dibujo como método de trabajo, el cual ha sido mayoritariamente desarrollado en espacios comunes significativos para la comunidad, como por ejemplo sedes vecinales y sindicatos. Estos son usualmente espacios cerrados que son cómodos y familiares $\mathrm{y}$, por tanto, favorecen el recuerdo y la conversación, pero que son riesgosos en el contexto pandémico. Creemos interesante avanzar en metodologías que impliquen recorrer el lugar, caminar juntos los espacios significativos. Estos ejercicios caminantes se han hecho generalmente en espacios urbanos como es el caso del Paseo de Jane y Transurbancia (Jiménez y Useros, 2016; Careri, 2002) - y constituye un desafío adaptarlas a la amplitud de los espacios no urbanos, diseñando métodos "transrurales", "transprediales", "transforestales" y "transmarinos" que pudieran ser recorridos a caballo, en bicicleta o lancha, y que favorezcan -en el movimiento y la visita- la reapreciación del espacio.

\section{5.- Reflexiones finales}

El uso de metodologías elaboradas sobre la base de la técnica de cartografía social nos ha permitido no solo investigar sobre el reconocimiento de la alteridad económica de los territorios, la defensa, creación y cuidado de comunes y sus gobernanzas territoriales, sino, además, promover una plataforma de reflexión acordada y colectiva.

El trabajo comienza en relación negociada entre el mundo académico y comunidades territoriales. Esto constituye un encuentro de mundos epistémicos: la academia existe y se construye en los estándares occidentales y científicos de producción de conocimiento, y se evalúa por estándares de publicación. Ambas características no solo no tienen sentido, sino que incluso violentan epistémicamente a algunas comunidades, generando como consecuencia el rechazo activo a la presencia de investigadores universitarios. Las comunidades, por su parte, entran a participar en estos procesos a partir de una variedad de motivaciones tales como visibilizar un conflicto, obtener insumos para procesos de negociación y lucha socioterritorial, visibilizar una iniciativa comercial o turística, u otros. La cuestión es, entonces, cómo negociar, integrar y balancear estas distintas expectativas e intereses. Ello implica un ejercicio de transparencia, generosidad y negociación entre los actores, el cual debe ser desarrollado desde el diálogo de saberes, horizontalidad y respeto mutuo. Muchas veces, esto implica que la academia se sume a las dinámicas políticas y económicas, y a los procesos de reflexión desarrollados por la comunidad. Pero también, en ocasiones, el proceso de investigación no se comienza siempre desde la iniciativa activa de la 
comunidad, sino que es el equipo investigador quien estimula en la comunidad el interés en desarrollar un proceso reflexivo que sea útil a sus propios horizontes políticos y de sentido. Como resultado de esta relación se generan cambios en ambas partes -la comunidad y la academia- generando procesos de transculturalidad y transvaloración (Todorov, 1991).

La cartografía social como método permite organizar y dar materialidad espacial y simbólica a la información, conocimiento, saberes, deseos, imaginaciones sobre los territorios. Cuando se trabaja en forma transdisciplinaria (entre distintas visiones académicas) e intercultural (entre distintas visiones de mundo), se logra integrar perspectivas heterogéneas para la reflexión, el análisis y la producción de conocimiento sobre los territorios. Al contar con un soporte físico que representa el territorio (el mapa) y herramientas (lápices, papeles, etiquetas, íconos) que permiten volcar sobre este los conocimientos, saberes y sentires, se genera un diálogo hablado, escrito, dibujado y, a veces, silencioso entre todos los participantes, pudiendo no solo volcar una experiencia individual a una colectiva, sino también explorar otras perspectivas de la experiencia, producir conocimiento y alternativas de acción que surgen en este encuentro de diferentes mundos.

Los ejercicios de cartografía social realizados fueron instancias de diálogo entre las comunidades y actores del ámbito académico, provocando tres procesos a partir del ejercicio de coproducción de conocimiento. Un primer proceso corresponde a la visualización de la comunidad y de sus propios elementos constitutivos, en este caso, prácticas de diversidad económica, los comunes, sus amenazas y sus acciones de gobernanza. Tanto el equipo investigador como la comunidad reconocen estos elementos, los analizan y los visibilizan. Ello permite revisitar lo cotidiano y dado por hecho para permitir, a través de una reflexión colectiva, resemantizar la realidad -sus problemáticas y posibilidades-, esto es, remirarla y nombrarla desde otras perspectivas.

El segundo efecto es la sorpresa colectiva. Tanto el equipo investigador como los actores se sorprenden con el reconocimiento de los comunes y las formas de gobernanza de la comunidad. La resemantización de las prácticas cotidianas, como prácticas de cuidado, conservación y transformación, dan nuevos sentidos a los territorios, desde categorías no pensadas antes y que, por lo tanto, adquieren otro valor. El equipo investigador también adquiere una nueva consciencia sobre sus supuestos e hipótesis -y también sobre los límites del conocimiento científico-, siendo transformadora tanto para las personas como para la investigación en sí.

Como resultado se genera un tercer efecto de transculturalidad y transvaloración, por los cuales la mirada del otro enriquece y transforma la propia mirada. Todos los participantes del proceso de investigación -actores del territorio y actores académicos- salen transformados del proceso, con aprendizajes significativos y cambios -a veces profundos- 
sobre la realidad territorial, sobre la propia subjetividad y también sobre la relación academia-comunidad.

Finalmente, esta revaloración propia de la comunidad se hace visible a otros grupos. Los cambios en los procesos de subjetividad y narrativa territorial fortalecen la identidad colectiva -el nosotros, el relato común, la memoria y el trayecto- permitiendo posicionar la comunidad con más fuerza con relación a otras comunidades y con relación a actores más poderosos. Se favorecen así procesos de conexión y solidaridad con otras comunidades (llevar a otros nuestra experiencia aprendida) y de promover procesos de replicabilidad y escalabilidad de los procesos documentados: inspirar otros procesos territoriales.

\section{6.- Bibliografía}

Andrade, H. y Santamaría, G. (1997). Cartografía Social para la planeación participativa. Memorias del Curso: Participación Comunitaria y Medio Ambiente. Bogotá, Colombia: Ministerio del Medio Ambiente e ICFES. Recuperado de http://www.beu.extension.unicen.edu.ar/xmlui/bitstream/handle/123456789/354/Andrade C ARTOGRAFIA SOCIAL PARTICIPATIVA.pdf?sequence=12amp;isAllowed =y

Butler, J. (1990). Gender Trouble. Routledge: London.

Callon, M. (2007). 'What does it mean to say that economics is performative?', en (eds) D. MacKenzie, F. Muniesa y L. Siu. Do Economists Make Markets? On the Performativity of Economics (pp. 311-357). Princeton: Princeton University Press.

Careri, F. (2002). Walkscapes. El andar como práctica estética. Barcelona, España: Editorial Gustavo Gili.

Carrasco Bengoa, C. (2001). La sostenibilidad de la vida ¿Un asunto de mujeres? Revista Mientras tanto, 82, 43-70.

Diez, J.M. y Chanampa, M.E. (2016). Perspectivas de la Cartografías Social, experiencias entre extensión, investigación e intervención social. Revista $+E, 6$, 84-94. Recuperado de https://bibliotecavirtual.unl.edu.ar/publicaciones/index.php/Extension/article/view/6316/928 $\underline{2}$

Escobar, A. (2017). Autonomía y diseño: la realización de lo comunal. Popayán: Tinta Limón.

Eskelinen, T.; Hirvilammi, T. y Venäläinen, J. (2020). Enacting Community Economies Within a Welfare State. MayFly Books. Recuperado de http://mayflybooks.org/wpcontent/uploads/2020/03/ECEWAWS_final_e-book.pdf 
Fals-Borda, O. (2009). Orígenes universales y retos actuales de la Investigación-Acción Participativa. En Rodríguez, J. (comp.) Desarrollo regional y planificación del territorio, Cuadernos de clase $N^{\circ}$ 2. Manizales: Universidad Autónoma de Manizales.

Gibson-Graham, J.K. (2006). Postcapitalist Politics. Minnesota: University of Minnesota.

Gibson-Graham, J.K.; Cameron, J. y Healy, S. (2017). Retomemos la economía: una guía ética para transformar nuestras comunidades. Bogotá: Editorial Pontificia Universidad Javeriana.

Habbeger, S. y Mancila, I. (2006). El poder de la Cartografía Social en las prácticas contrahegemónicas o La Cartografía Social como estrategia para diagnosticar nuestro territorio. Revista Araciega, 14.

Hardin, G. (1968). La tragedia de los comunes. Science, 162(37), 1243-1248.

Hardt, M. y Negri, A. (2011). Commonwealth: El Proyecto De Una Revolución Del Común. Madrid: Ediciones Akal.

Harvey, D. (2004). El nuevo imperialismo: acumulación por desposesión. Socialist register.

Jiménez, S. y Useros, A. (2016) El Paseo de Jane: Tejiendo Redes a Pie de Calle. España: Modernito Books.

Laval, C. y Dardot, P. (2014). Común: ensayo sobre la revolución en el siglo XXI. Barcelona: Editorial Gedisa.

Montoya, V.; Sánchez, A. y Ospina, C. (2014). Andar dibujando y dibujar andando: Cartografía social y producción colectiva de conocimientos. Nómadas, 40, 190-205. Recuperado de https://www.redalyc.org/articulo.oa?id=105131005013

Ostrom, E. (1992). Governing the Commons: The Evolution of Institutions for Collective Action, Cambridge: Cambridge University Press.

Pérez, D.A.A. (2011). Hermeneutics and research methods of Social Science. Estudios de Filosofía, (44), $7 . \quad$ Recuperado de http://www.scielo.org.co/scielo.php?script=sci arttext\&pid=S012136282011000200002\&lng=en\&tlng=en.

Pérez Orozco, A. (2004). Estrategias feministas de deconstrucción del objeto de estudio de la economía. Foro Interno, 4, 87-117.

Todorov, T. (1991). Nosotros y los otros. Reflexión sobre la diversidad humana. Ciudad de México: Siglo Veintiuno Editores. 